Urologe $2020 \cdot 59: 1067-1075$

https://doi.org/10.1007/s00120-020-01206-9

Online publiziert: 28. April 2020

(c) Der/die Autor(en) 2020

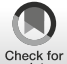

M. Neuberger ${ }^{1}$ C. Wei $\beta^{2} \cdot$ N. Westhoff ${ }^{1}$ T. S. Worst ${ }^{1} \cdot$ M. S. Michel' $\cdot$ J. von Hardenberg ${ }^{1}$

${ }^{1}$ Klinik für Urologie und Urochirurgie, Medizinische Fakultät Mannheim, Universität Heidelberg Mannheim, Deutschland

${ }^{2}$ Abteilung für Medizinische Statistik, Biomathematik und Informationsverarbeitung der Medizinischen Fakultät Mannheim, Universität Heidelberg, Mannheim, Deutschland

\section{Urologische Forschung in Deutschland}

\section{Eine retrospektive, longitudinale Beobachtungsstudie}

review veröffentlichten Forschungsarbeiten präsentiert werden. Dies kann eine Massenproduktion von Abstracts ohne Ziel nachfolgender Vollpublikation anstoßen [25]. So konnten Scherer et al. 2018 in einer Metaanalyse von 425 Arbeiten (307.028 Abstracts) zu Veröffentlichungen in den Feldern Grundlagenwissenschaften und Medizinische Forschung eine nachfolgende Vollpublikationsrate von $37,3 \%$ ermitteln [23]. Diese liegt signifikant niedriger als die vorangegangene, im Jahr 2007 veröffentlichte Auswertung desselben Teams (Publikationsrate 44,5\%; 29.729 Abstracts; [26, 27]). Zudem konnte ein Publikationsbias aufgezeigt werden: Positive Ergebnisse führen signifikant häufiger zu einer Publikation als negative [27]. Zusätzlich besteht trotz Peer-review-Verfahren die Gefahr eines Reviewer-Bias [25].

Die vorliegende Untersuchung stellt zum einen durch die Ermittlung der Anzahl und des Journals der nachfolgenden Vollpublikation eine Qualitätsanalyse dar. Zum anderen soll sie positive und negative Trends identifizieren, die als Ansatzpunkte für Bestrebungen zur Aufrechterhaltung und Steigerung der Qualität der urologischen Forschung in Deutschland dienen sollen, um diese international konkurrenzfähig zu halten. Analyse des Kongresses 2016 sowie der longitudinale Vergleich mit den beiden bereits analysierten Kongressen hinsichtlich Trends in der urologischen Forschung in Deutschland.

\section{Einleitung}

Wissenschaft ist die treibende Kraft menschlichen Fortschritts. Kongresse gelten traditionell als prägendes Element des akademischen, sozialen und beruflichen Lebens medizinischer Fachdisziplinen [17] und dienen dem wissenschaftlichen Austausch und der Vernetzung. Es ist bekannt, dass die wissenschaftliche Qualität eines Kongresses von der Qualität seiner Beiträge abhängt [11]. Wissenschaft ist in der Lage sich durch Analyse der eigenen Methoden, durch gegenseitige Evaluation und Reproduzierbarkeit selbst zu reflektieren [18].

Ein Kritikpunkt an Kongressen ist, dass häufig Abstracts und keine peer-

\section{Tab. 1 Übersicht über die aus den Kongressabstracts erfassten Parameter}

\begin{tabular}{ll} 
Organ & Studiendesign \\
\hline Prostata & Prospektiv/retrospektiv \\
Niere & Monozentrisch/multizentrisch \\
Ableitende Harnwege & Präklinisch-experimentell \\
Hoden & Statistik \\
Penis & Angabe von $p$-Werten im Abstract als Indikator \\
Anderes Organ & für durchgeführte statistische Analysen \\
Kein Organ & Kooperationen \\
Onkologie & Intern \\
Onkologischer Kontext & National \\
Klinischer Bereich & International \\
Therapeutisch & Industrie \\
Diagnostisch & Forschungseinrichtung \\
& Technik \\
Studiengröße & Beschreibung einer Technik \\
Anzahl Patienten/Patientenproben &
\end{tabular}


Tab. 2 Anzahl der Abstracts nach Organsystemen

\section{Organsysteme}

Alle Abstracts $(n=342)$

Ableitende Harnwege ${ }^{\mathrm{a}}, n(\%)$

Abstracts mit onkologischem Bezug $(n=211)$

Prostata, $n(\%)$

$139(40,6)$

Prostata, $n$ (\%)

$99(46,9)$

Niere, $n(\%)$

$116(33,9)$

Ableitende Harnwege, $n$ (\%)

$54(25,6)$

Penis, $n(\%)$

$46(13,5)$

Niere, $n(\%)$

$36(17,1)$

Hoden, $n(\%)$

$18(5,3)$

Penis, $n$ (\%)

$12(5,7)$

$17(5,0)$

Hoden, $n(\%)$

$10(4,7)$

Andere Organe, $n$ (\%)

$5(1,5)$

Andere Organe, $n(\%)$

$0(0)$

aUrothel in Nierenbecken, Harnleiter, Harnblase und Harnöhre sowie Urolithiasis

Tab. 3 Top-5-Journals insgesamt und Top 5 der Open-access-Journals, in denen Kongressabstracts aus dem Jahr 2016 veröffentlicht wurden

\begin{tabular}{|c|c|c|c|c|c|c|c|}
\hline \multirow[b]{2}{*}{ Rang } & \multicolumn{3}{|l|}{ Alle Journals } & \multicolumn{4}{|l|}{ Open-access-Journals } \\
\hline & Journal & $n(\%)$ & IF & Rang (fortlaufend) & Journal & $n(\%)$ & IF \\
\hline 1 & World J Urol & $16(8,9)$ & 2,77 & \multirow[t]{2}{*}{$1(12)$} & PLoS One & $3(1,7)$ & 2,78 \\
\hline 2 & Urol Int & $14(7,8)$ & 1,54 & & Sci Rep & $3(1,7)$ & 4,12 \\
\hline 3 & Oncotarget $^{\mathrm{a}}$ & $11(6,1)$ & 5,19 & \multirow[t]{2}{*}{$2(18)$} & Int Neurourol J & $2(1,1)$ & 1,81 \\
\hline 4 & BJU Int & $11(6,1)$ & 4,47 & & Front Pharmacol & $2(1,1)$ & 4,23 \\
\hline 5 & Urologe & $7(3,9)$ & 0,35 & $3(29)$ & Alle anderen & $1(0,6)$ & - \\
\hline
\end{tabular}

\section{Design und Untersuchungs- methoden}

\section{Auswahl der Abstracts}

Analysiert wurden 342 Abstracts des DGU-Kongresses 2016. Abstracts aus den Sessions „Filmbeiträge“, „Geschichte der Medizin“ und „Fallbeiträge“ wurden analog zur Vorarbeit ausgeschlossen. Die Daten der analysierten Abstracts der Kongresse von $2002(n=352)$ und 2009 $(n=380)$ standen zur Verfügung [29]. Durch die Auswahl des Kongresses 2016 wurde einerseits ein konstanter Abstand zwischen den analysierten Kongressen gehalten und andererseits, analog zur vorangegangenen Analyse, ein Follow-up von mindestens 3 Jahren gewährleistet.

\section{Erfasste Parameter}

Die erfassten Parameter zeigt - Tab. 1. In den Rubriken „Organ“, „Onkologie“, „klinisches Gebiet" und „Studiendesign“ wurden Einzelzuordnungen getroffen, wohingegen beim Kooperationsverhalten Mehrfachnennungen möglich waren. Die Zusammenarbeit innerhalb verschiedener Abteilungen eines Krankenhauses bzw. verschiedener Kliniken oder Institute einer Fakultät wurde als ,interne Kooperation" gewertet. Der $p$-Wert diente als Surrogatparameter für eine statistische Analyse. Weitere Parameter waren aus den Abstracts hervorgehende Publikationen inklusive Veröffentlichungsdatum und Journal. Letzteres wurde bezüglich seiner Zugehörigkeit zum Fachgebiet Urologie bewertet. Dieser Parameter wurde für die Kongresse 2002 und 2009 nacherfasst.

\section{Identifikation nachfolgender Publikationen}

Die Auswahl der Abstracts des DGUKongresses 2016 (Abstract-Deadline 13.03.2016) gewährleistete einen Nachbeobachtungszeitraum von 3 Jahren. Zur Identifizierung einer nachfolgenden Publikation gingen wir methodisch wie in der vorangegangenen Veröffentlichung vor [29]. Der „impact factor“ aus dem Jahr der Publikation wurde aus dem „Journal Citation Report" von Clarivate Analytics (Clarivate ${ }^{\mathrm{m}}$, Boston, Massachusetts, USA) übernommen.

\section{Statistik}

Es erfolgte eine deskriptive Datenanalyse. Der longitudinale Vergleich erfolgte für kategoriale Variablen mittels $\chi^{2}$ - und Trendtest nach Cochran-Armitage. Für quantitative Merkmale wurden der Kruskal-Wallis- und der Mann-Whitney-UTest herangezogen. Sämtliche Tests wurden mit SAS ${ }^{\circledR}$ (SAS Institute Inc., Cary, North Carolina, USA) durchgeführt und $p$-Werte $<0,05$ als signifikant gewertet.

\section{Ergebnisse}

\section{Kongress 2016}

Die in den 342 Abstracts am häufigsten vertretenen Organe bzw. Organsysteme insgesamt und onkologisch sind in - Tab. 2 dargestellt. Die meisten Abstracts konnten dem klinischen Gebiet „Therapie chirurgisch“ (38,0\%), gefolgt von „Diagnostik“ (26,9\%) und „präklinisch-experimentell“ (22,8\%) zugeordnet werden. Einen Überblick über das Kooperationsverhalten zeigt $\bullet$ Abb. 1. $277(81 \%)$ der 342 Abstracts konnten einem mono- oder multizentrischen, $211(61,7 \%)$ einem pro- oder retrospek- 
Hier steht eine Anzeige.

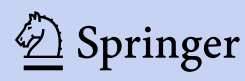


Urologe 2020 -59:1067-1075 https://doi.org/10.1007/s00120-020-01206-9

(c) Der/die Autor(en) 2020

M. Neuberger · C. Weiß · N. Westhoff · T. S. Worst · M. S. Michel · J. von Hardenberg

\section{Urologische Forschung in Deutschland. Eine retrospektive, longitudinale Beobachtungsstudie}

\section{Zusammenfassung}

Hintergrund und Ziel der Arbeit. Der

Jahreskongress der Deutschen Gesellschaft für Urologie (DGU) spiegelt die urologische Forschungslandschaft im deutschsprachigen Raum wider. Ziel war es, durch die longitudinale Analyse der Kongressabstracts und hervorgehenden Vollpublikationen Trends aufzudecken.

Material und Methoden. Es erfolgte die systematische Analyse der Kongressabstracts des Jahreskongresses 2016 auf Inhalt, Studiendesign, Kooperationen, hervorgehenden Vollpublikationen sowie der veröffentlichenden Journalen. Hiernach erfolgte der Vergleich mit den Kongressen 2002 und 2009. Statistische Berechnungen erfolgten per $\chi^{2}-$, Mann-Whitney-U-, CochranArmitage- und Kruskal-Wallis-Test. Ergebnisse. Auf den Kongressen 2002, 2009 und 2016 wurden 1073 Abstracts präsentiert. Abstracts zu Prostataerkrankungen ( $24,2 \%$, $29,7 \%, 34,0 \% ; p=0,0043)$, onkologische Abstracts (50,6\%, 57,9\%, 61,7\%; $p=0,003)$, multizentrische Studien (18,3\%, 28,6\%, $34,3 \% ; p<0,0001)$ und Kooperationsprojekte $(55,6 \%, 62,9 \%, 70,5 \%, p<0,0001)$ nahmen zu, experimentelle $(29,0 \%, 33,2 \%, 22,8 \%$; $p=0,009)$ und prospektive Arbeiten $(62,1 \%$, $42,0 \%, 36,0 \% ; p<0,0001)$ ab. Statistische Analysen (18,4\%, 14,7\%, 41,2\%; $p<0,0001)$ und der "impact factor" nachfolgender Vollpublikationen $(2,08,3,42,4,42 ; p<0,0001)$ stiegen. 2016 erfolgten $11,2 \%$ dieser
Vollpublikationen "open access". Die Rate veröffentlichter Abstracts betrug 49,1\%, $56,3 \%$ und $52,3 \%(p=0,15)$.

Schlussfolgerung. Die nationale und internationale Vernetzung der urologischen Forschungsgemeinschaft nimmt zu, prospektive Studien werden weniger präsentiert. Die Rate aus DGU-Abstracts hervorgehender Vollpublikationen zeigt sich über die drei Kongresse auf hohem Niveau. Die Veröffentlichungsrate in Open-accessJournalen ist bisher gering.

Schlüsselwörter Deutsche Gesellschaft für Urologie · Publikationserfolg · Studiendesign · Kooperation · Metaforschung

\section{Urological research in Germany. A retrospective, longitudinal observational study}

\section{Abstract}

Background and objectives. The congress of the German Society of Urology reflects urologic research in German-speaking countries. The objective was to identify trends by analyzing the congress' abstracts and following full publications longitudinally. Materials and methods. The abstracts of the 2016 congress were systematically analyzed regarding content, study design, cooperation, following full publications and journals which they were published in. Thereafter, the 2016 congress was compared to the 2002 and 2009 congresses. Statistical analysis included $x^{2}$ Mann-Whitney U-, Cochran-Armitage-, and Kruskal-Wallis test.
Results. A total of 1073 abstracts were presented at the 2002, 2009, and 2016 congresses. We found an increase in abstracts regarding prostate disease $(24.2 \%, 29.7 \%$, and $34.0 \% ; p=0.0043)$, oncological abstracts $(50.6 \%, 57.9 \%$, and $61.7 \% ; p=0.003)$, multicenter studies (18.3, 28.6, and 34.3\%; $p<0.0001)$ and cooperation $(55.6 \%, 62.9 \%$, and $70.5 \%, p<0.0001)$. Experimental $(29.0 \%$, $33.2 \%$, and $22.8 \% ; p=0.009$ ) and prospective studies $(62.1 \%, 42.0 \%$, and $36.0 \% ; p<0.0001)$ declined. Abstracts including statistical analysis $(18.4 \%, 14.7 \%$, and $41.2 \% ; p<0.0001)$ and the impact factor of following full publications $(2.08,3.42,4.42 ; p<0.0001)$ rose. In $2016,11.2 \%$ of those full publications were published Open Access. The publication rates of the presented abstracts were $49.1 \%, 56.3 \%$, and $52.3 \%$, respectively $(p=0.15)$.

Conclusions. National and international networking of the urological research community has increased. Presentation of prospective studies has declined. The rate of peer-reviewed full publications following the DGU abstracts remains at a stable high level over the three congresses. The publication rate in Open Access journals is low.

\section{Keywords}

German Society of Urology · Publication success - Study design · Cooperation · Research on research tiven Design zugeordnet werden. 36,0\% der zuordenbaren Abstracts waren prospektiv, 34,3\% multizentrisch. Unter den 76 prospektiven Arbeiten waren 55,3\% monozentrisch und 44,7\% multizentrisch. Die Patientenanzahl pro Abstract betrug im Median 131. Die höchsten Impakt-Faktoren (IF) wurden in den Organfeldern „Prostata“ (Median: 3,89, Range: 0,3-33,9) und „Penis“ (Median: 2,85, Range: 1,27-16,27) erzielt. Aus 179 (52,3\%) der analysierten Abstracts des Kongresses 2016 gingen Vollpublikationen in Peer-review-Journals hervor. Für diese lag der IF im Mittel bei 4,41 $\pm 5,62$ (Median 2,86, Range: 0,24-47,8). $56 \%$ $(n=101)$ der resultierenden Publikationen erfolgte in einem urologischen Journal, eine Open-access-Publikation in $11,2 \%(n=20)$. - Tab. 3 zeigt die fünf Journale insgesamt sowie „open access“ mit den meisten Veröffentlichungen.

\section{Longitudinale Analyse 2002, 2009} und 2016

Im longitudinalen Vergleich der 1073 Abstracts der Kongresse 2002, 2009 und
2016 nahmen Abstracts zum Thema „Prostata“ (24,2\% vs. $29,7 \%$ vs. $34,0 \%$; $p=0,004)$ sowie onkologische Themen (50,6\% vs. $57,9 \%$ vs. $61,7 \% ; p=0,003$ ) zu. - Abb. 2a zeigt die Entwicklung aller onkologischen Abstracts. Kooperationsprojekte allgemein $(55,6 \%$ vs. $62,9 \%$ vs. $70,5 \%, p \leq 0,0001)$, innerhalb einer Klinik/Fakultät (2002 und 2009 28,7\% vs. $201638,3 \% ; p=0,0068)$ sowie auf nationaler $(23,6 \%$ vs. $31,3 \%$ vs. $40,6 \%$, $p<0,0001)$ und internationaler Ebene ( $13,6 \%$ vs. $14,7 \%$ vs. $19,3 \%, p=0,042$ ) nahmen ebenfalls zu (s. • Abb. 2b). Die 


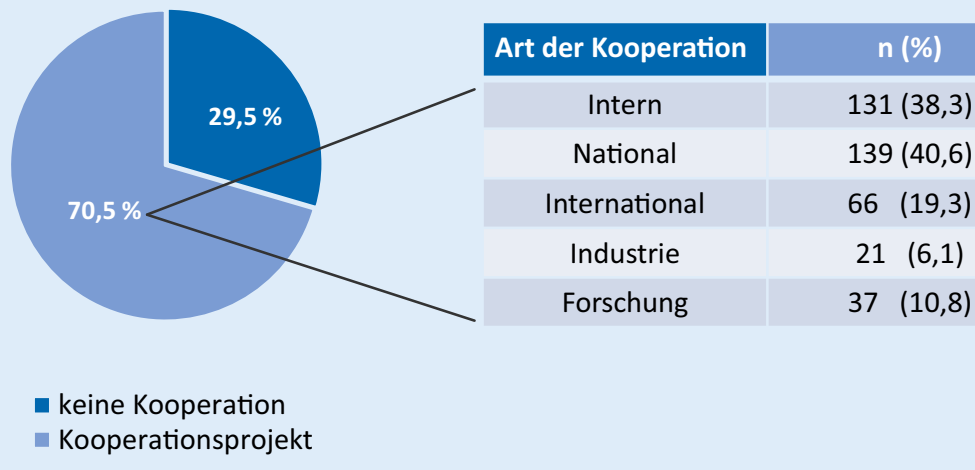

Abb. 1 A Verteilung der Kooperationen der Kongressabstracts 2016 (Mehrfachnennung möglich)

Zahl experimenteller Arbeiten zeigte sich 2016 signifikant niedriger $(p=0,009$, s. Abb. 2 c [ $29,0 \%$ vs. $33,2 \%$ vs. $22,8 \%])$. Der Anteil diagnostischer Arbeiten zeigt signifikante Unterschiede $(29,6 \%$ vs. $18,3 \%$ vs. $34,9 \%, p<0,0001)$, jedoch keinen Trend. Hinsichtlich Abstracts, die primär eine Technik beschreiben oder eine klinische Fragestellung beinhalten, zeigen sich teilweise signifikante Unterschiede zwischen einzelnen Kongressen, jedoch ohne erkennbaren (Aufwärts- oder Abwärts-)Trend (nicht dargestellt). Der Anteil retrospektiver Arbeiten $(37,8 \%$ vs. $57,9 \%$ vs. $64,0 \%$; $p<0,0001)$ und multizentrischer Studien $(18,3 \%$ vs. $28,6 \%$ vs. $34,3 \% ; p<0,0001)$ nahm ebenfalls zu (s. $\bullet$ Abb. 2d). Zudem stieg die Anzahl eingeschlossener Patienten im Vergleich zu den vorherigen Kongressen $(p<0,0001)$. Im Vergleich zum Kongress 2002 zeigte sich ein Anstieg des ,impact factors“ (IF) (2002: 2,08 vs. 2009: 3,$42 ; p<0,0001$ bzw. vs. 2016: 4,42; $p<0,0001)$ sowie eine schnellere Veröffentlichung (2002: 19,03 Monate vs. 2016: 13,25 Monate, $p<0,0045)$. Die Anzahl an Abstracts mit $p$-Wert (18,4\% vs. $14,7 \%$ vs. $41,2 \%, p<0,0001)$ nahm zu. Die Publikationsrate, gemessen an aus Kongressabstracts hervorgehenden Publikationen in Peer-review-Journals, blieb ohne signifikante Veränderungen ( $49,1 \%$ vs. $56,3 \%$ vs. $52,3 \%, p=0,15$, s. - Abb. 2e). - Abb. 2f zeigt die Entwicklung publizierter onkologischer Abstracts. Es lässt sich eine Zunahme an Veröffentlichungen in nicht-urologischen Journals beobachten ( $26,6 \%$ vs. $32,2 \%$ vs. $43,6 \%, p=0,0008)$.

\section{Diskussion}

Die Abnahme des Anteils nicht-onkologischer Themen bleibt kritisch zu beobachten. Das Ziel urologischer Forschung sollte die beständige Weiterentwicklung des Faches in allen Bereichen sein, weshalb nicht-onkologische Themen im Sinne des Patientenwohls nicht ins Hintertreffen geraten sollten. Andere Bereiche, wie z.B. die Urolithiasis und die gutartige Prostatavergrößerung, gelten teilweise als nicht ausreichend gewürdigt. Eine aktuelle und auf Deutschland beschränkte Abfrage auf ClinicalTrials.gov registrierter Studien ergab für den Suchbegriff „Urolithiasis“ eine und für „BPH“ 34 Studien. Dem gegenüber stehen 222 registrierte Studien für „Prostate Cancer“ sowie 113 Studien zum Thema „Renal Cell Carcinoma" [3]. Auch innerhalb onkologischer Themen gibt es Unterschiede der als nachfolgende Vollpublikation publizierten Abstracts. Esfällt eine signifikante Abnahme im Bereich Urothelkarzinom (ableitende Harnwege) über die drei analysierten Kongresse auf. Dies deckt sich mit einer 2013 erschienenen deskriptiven Analyse von Kunath et al. mit dem Titel "Bladder cancer - the neglected tumor" [19] und wird auch in der geringen Anzahl in Deutschland registrierter Studien zum Thema Urothelkarzinom (37 Studien) deutlich [3].

Die Bedeutung interdisziplinärer Forschung nimmt zu. Dies spiegelt sich im Anstieg an Veröffentlichungen in nicht-urologischen Journals wider und könnte u.a. durch den zuletzt zunehmenden Anteil an Abstracts zum Thema
„Diagnostik“ bedingt sein, da z. B. viele Artikel zur Weiterentwicklung der Schnittbildgebung in radiologischen/ nuklearmedizinischen Journals publiziert wurden.

Die Anzahl an nationalen und internationalen Kooperationen im medizinischen Bereich hat in den letzten 20 bis 30 Jahren zugenommen [12, 32]. Nicht nur die Anzahl an Kooperationsprojekten insgesamt, sondern insbesondere die Zunahme internationaler Kooperationsprojekte und multizentrischer Arbeiten unterstreicht die gute Vernetzung deutscher Urologen. Durch die Gründung der German Society of Residents in Urology (GeSRU) Academics Anfang 2014 besteht die Möglichkeit für eine frühe Vernetzung von jungen Urologen in Deutschland über die eigene Institution hinaus. Im Sinne einer besseren Vernetzung könnte man zusätzlich jungen Wissenschaftlern auf dem Weg zur Habilitation die Rotation in Arbeitskreise der DGU ermöglichen. Der so gewonnene Wissensaustausch und die Vernetzung könnten zur Wahrung und Steigerung der Forschungsqualität beitragen.

Die zunehmende Vernetzung sowie die bessere Nutzung von Synergien können zukünftig zu einer weiteren Steigerung der Forschungsqualität führen. So konnte gezeigt werden, dass Veröffentlichungen, die aus internationalen Kooperationen resultieren, in Journals mit höherem IF erscheinen und häufiger zitiert werden [5]. Andererseits sind Kooperationen notwendig, um die zunehmend komplexen Herausforderungen und Möglichkeiten in der Forschung zu bewältigen [15]. Nationale Kooperationen konnten neben der Angabe von Statistik und einem prospektiven oder experimentellen Studiendesign in der dieser Arbeit vorangegangenen Analyse als prädiktive Faktoren für einen nachfolgenden Publikationserfolg identifiziert werden [29]. Die Wichtigkeit der Vernetzung für einen Publikationserfolg wird durch weitere Studien belegt [9].

$\mathrm{Zu}$ beobachten ist die signifikante $\mathrm{Ab}$ nahme experimenteller Arbeiten. Mit der Initiierung der Ferdinand EisenbergerForschungsstipendien für Mediziner im Jahr 2010 und der Wolfgang LutzeyerStipendien für naturwissenschaftliche 

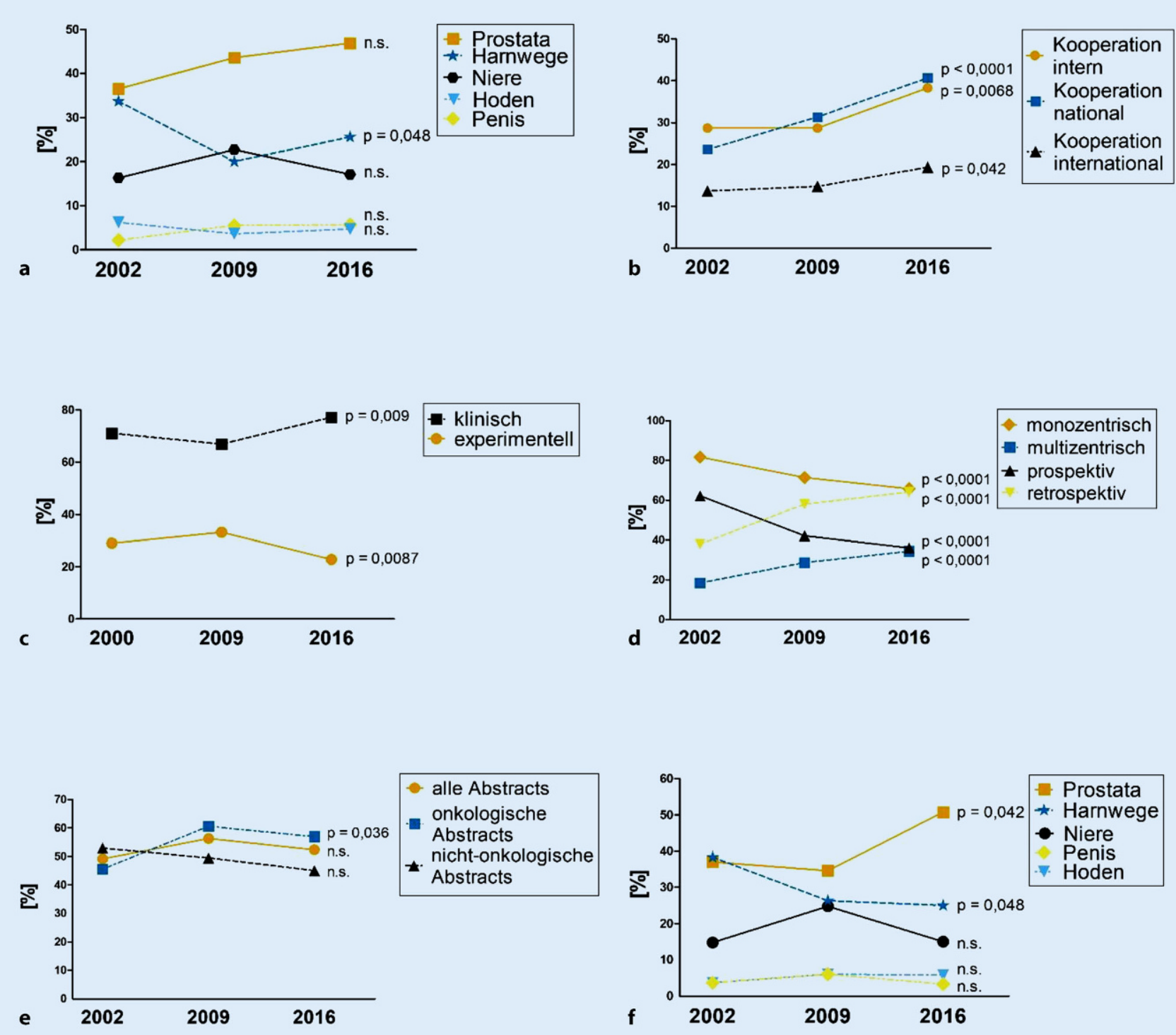

Abb. $2 \Delta$ Longitudinaler Vergleich der auf den Kongressen präsentierten onkologischen Abstracts (a), des Kooperationsverhaltens (b), experimenteller bzw. klinischer Arbeiten (c), der Studiendesigns (d), aus den Abstracts hervorgehender, peer-reviewed publizierter Arbeiten (e) sowie der peer-reviewed publizierten onkologischen Abstracts (f)

Forscher im Jahr 2018 hat die DGU bereits zwei wichtige Schritte zur Förderung des wissenschaftlichen Nachwuchses und damit zur Zukunftsgestaltung der experimentellen Forschungslandschaft unternommen. Zudem existieren Forschungsförderungen der Deutschen Forschungsgemeinschaft (DFG) z.B. im Rahmen von Forschungsstipendien, dem Emmy Noether- und dem Walter Benjamin-Programm. Gerade die wichtige translationale Forschung, wie z.B. an prädiktiven und prognostischen Biomarkern, sollte nicht nur durch Grundlagenwissenschaftler erfolgen.
In der Analyse konnte eine Abnahme an Abstracts zu prospektiven Studien bei gleichzeitig mehr retrospektiven Studien feststellt werden. Prospektive Studien sowie deren Reviews und Metaanalysen liefern die höchsten Evidenzlevel und stellen die Grundlage für evidenzbasierte Medizin dar [4]. Die Entwicklung von Leitlinien basiert weitestgehend auf prospektiven Studien. Eine konsequente Registrierung prospektiver klinischer Studien stellt einen wertvollen Baustein auf dem Weg zu unvoreingenommenerem (,unbiased“) Veröffentlichen dar [30]. Aufgrund dieser Tatsa- che wurde ein Passus in die Deklaration von Helsinki aufgenommen, dass jede klinische Studie in einer öffentlich zugänglichen Datenbank registriert sein muss, bevor der Einschluss des ersten Patienten erfolgt [2]. Dies hatte die Etablierung von Datenbanken wie Clinical Trials.gov zur Folge. Zum einen besteht jedoch eine geringe Compliance bezüglich der Veröffentlichungen der Studienergebnisse von teilweise 13-39\% [1], zum anderen konnte gezeigt werden, dass bis zu $30 \%$ der prospektiven Studien, die in High-impact-Journalen veröffentlicht wurden, nicht registriert waren [14]. Ten- 
denziell besitzen retrospektiv erhobene Daten eine geringere Aussagekraft und können zu Ergebnisverzerrungen führen [20, 28]. Aus den Analysen kann jedoch nicht beurteilt werden, ob prospektive Studien z. B. auf anderen Kongressen (ASCO GU, ASCO, EAU) vorgestellt werden oder ob die Anzahl an im deutschsprachigen Raum initiierten prospektiven urologischen Studien wirklich abnimmt. Weitere Analysen sind hier notwendig.

Die Publikationsrate von Abstracts als nachfolgende Vollpublikationen in einem Peer-review-Journal liegt über die analysierten Kongresse konstant hoch und im internationalen Vergleich urologischer Kongresse für DGU-Abstracts mit am höchsten (• Tab.4). Dies spricht für die Qualität der urologischen Forschung im deutschsprachigen Europa.

Eine Open-access-Veröffentlichung erfolgte in $11,2 \%$. Im Vergleich $\mathrm{zu}$ aktuellen Schätzungen und Zahlen ist dies deutlich unterdurchschnittlich [23]. In einer 2018 publizierten repräsentativen
Analyse wurden 27,9\% aller Artikel, die in Journals mit DOI (digitaler Objektbezeichner) veröffentlicht wurden, als „open access“ identifiziert [23]. Eine im Rahmen derselben Arbeit über die Rechercheoberfläche „Web of Science“ durchgeführte Analyse verschiedener Fachbereiche ergab für die „klinische Medizin“ einen Anteil an Open-accessArtikeln von nahezu $50 \%$ [23]. Eine Befragung der Kongressteilnehmer des DGU zum Thema Open-access-Publishing könnte mögliche Gründe aufzeigen und Verbesserungspotenziale erkennbar machen.

Zur Aufrechterhaltung der Forschungsqualität könnte eine zusätzliche Kongresssektion eingeführt werden, in der veröffentlichte Originalarbeiten diskutiert werden. Somit wäre zum einen eine Wissensmitnahme von abgeschlossenen und peer-reviewten Projekten gewährleistet. Zum anderen ist die Diskussion eines erfolgreichen Projekts ggf. Erfolg versprechender hinsichtlich sich hieraus ergebender Anschlussarbeiten.
Zudem könnte eine zusätzliche Kongresssession mit dem Titel „Triff Herausgeber und Reviewer" mehrere Punkte adressieren: Woran erkennt man ein unseriöses Journal? Worauf sollte man beim Erstellen einer Publikation und beim $\mathrm{Pu}$ blizieren achten?

\section{Limitationen}

Teilweise präsentierten Abstracts nationale Daten aus größeren, prospektiven, internationalen Studien (z. B. der „European Randomized Study of Screening for Prostate Cancer"). Die jeweiligen Autoren waren mit Publikationen im Zusammenhang zu den internationalen Studien mehrfach in MEDLINE gelistet. Jedoch wurde die Fragestellung des Abstracts nicht in der Veröffentlichung aufgegriffen. Daraufhin wurde der Abstract ohne Publikationserfolg gewertet.

Möglicherweise sind nicht alle nachfolgenden Vollpublikationen erfasst, insbesondere falls es zu starken Änderungen im Titel oder sowohl der Erst- als

Hier steht eine Anzeige. 
Originalien

Tab. 4 Internationaler Vergleich von Publikationsraten urologischer Kongresse

Kongress Jahr

American Urological Association

1998

1999

Aus präsentierten Ab-

stracts hervorgehende

Vollpublikationen, $\boldsymbol{n}(\%)$

1999
2000

$543(42,5)$

$570(37,8)$

$875(55,0)$

British Association of Urological

$2001+2002$

Surgeons

Deutsche Gesellschaft für

Urologie

2002

2009

2016

European Association of Urology

International Continence Mee-

ting

Korean Urological Association

Société Internationale

d'Urologie

Urological Brazilian Meeting

$2000+2001$

2003

$2006+2007$

$2002+2004$

$142(31,6)$

$173(49,2)$

$214(56,3)$

$179(52,3)$

$666(47,3)$

$77(61,6)$

$421(41,6)$

Referenz

Urological Society of Australia

2003

$415(22,1)$

$129(39,0)$

2005-2009 $183(29,8)$

$225(20,5)$

World Congress of Endourology $\quad 2001+2002$

auch Letztautoren gab. Durch die getrennte und kombinierte Suche der Erstund Letztautoren sowie Screening aller der jeweiligen Erst- und Letztautoren seit 2016 veröffentlichen und MEDLINE-gelisteten Publikationen sind wir jedoch zuversichtlich, dass wir den Großteil der Vollpublikationen erfasst haben.

Durch das retrospektive Design der Studie und den begrenzten Follow-upZeitraum von ca. 3,25 Jahren entsteht ein Selektionsbias. Internationale, prospektive oder experimentelle Arbeiten brauchen evtl. länger bis aus ihnen eine Publikation hervorgeht. Abstracts zu präklinisch-experimentellen Arbeiten könnten Daten zu einem frühen Zeitpunkt präsentiert haben, aus welchen erst im mittel- bis langfristigen Verlauf erfolgreiche Publikationen hervorgehen oder die Grundlage für erfolgreiche Forschung entsteht. Ein weiter gefasster Nachverfolgungszeitraum könnte diesen Bias reduzieren. Diese Arbeit analysierte lediglich von der DGU zum Kongress zugelassene/akzeptierte Abstracts, was ebenfalls zu einem Selektionsbias beitragen kann.

\section{Fazit für die Praxis}

- Die Anzahl an internen, nationalen und internationalen Kooperationen nimmt zu und unterstreicht die gute Vernetzung der deutschen Urologie. Retrospektive Arbeiten nehmen weiter zu, experimentelle und prospektive Arbeit ab, obwohl bei zunehmender Abbildung der Medizin in Leitlinien mehr prospektive Studien notwendig sind. Zur Aufrechterhaltung der hohen Forschungsqualität sollte diesen Trends entgegengewirkt und die Vernetzung junger forschender Urologen weiter gefördert werden.

- Die Rate der aus den Abstracts hervorgehenden Vollpublikationen zeigt sich in der longitudinalen Analyse stabil hoch und unterstreicht die Qualität des DGU-Jahreskongresses.

\section{Korrespondenzadresse

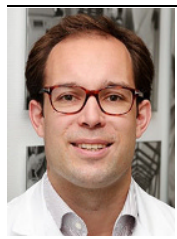 \\ PD Dr. med. J. von \\ Hardenberg \\ Klinik für Urologie und \\ Urochirurgie, Medizinische \\ Fakultät Mannheim, \\ Universität Heidelberg \\ Theodor-Kutzer-Ufer 1-3, \\ 68167 Mannheim, \\ Deutschland \\ jost.vonhardenberg@ \\ medma.uni-heidelberg.de}

Danksagung. Wir danken Herrn Olaf Kurpick für die Bereitstellung der Abstracts der Kongresse der DGU aus den Jahren 2002, 2009 und 2016.

Funding. Open Access funding provided by Projekt DEAL.

\section{Einhaltung ethischer Richtlinien}

Interessenkonflikt. M. Neuberger, C. Weiß, N. Westhoff, T.S. Worst, M.S. Michel und J. von Hardenberg geben an, dass kein Interessenkonflikt besteht.

Für diesen Beitrag wurden von den Autoren keine Studien an Menschen oder Tieren durchgeführt. Für die aufgeführten Studien gelten die jeweils dort angegebenen ethischen Richtlinien.

Open Access. Dieser Artikel wird unter der Creative Commons Namensnennung 4.0 International Lizenz veröffentlicht, welche die Nutzung, Vervielfältigung, Bearbeitung, Verbreitung und Wiedergabe in jeglichem Medium und Format erlaubt, sofern Sie den/die ursprünglichen Autor(en) und die Quelle ordnungsgemäß nennen, einen Link zur Creative Commons Lizenz beifügen und angeben, ob Änderungen vorgenommen wurden.

Die in diesem Artikel enthaltenen Bilder und sonstiges Drittmaterial unterliegen ebenfalls der genannten Creative Commons Lizenz, sofern sich aus der Abbildungslegende nichts anderes ergibt. Sofern das betreffende Material nicht unter der genannten Creative Commons Lizenz steht und die betreffende Handlung nicht nach gesetzlichen Vorschriften erlaubt ist, ist für die oben aufgeführten Weiterverwendungen des Materials die Einwilligung des jeweiligen Rechteinhabers einzuholen.

Weitere Details zur Lizenz entnehmen Sie bitte der Lizenzinformation auf http://creativecommons.org/ licenses/by/4.0/deed.de.

\section{Literatur}

1. Anderson ML, Chiswell K, Peterson ED et al (2015) Compliance with results reporting at ClinicalTrials.gov. N Engl J Med 372:1031-1039

2. World Medical Association (2009) Declaration of Helsinki. Ethical principles for medical research involving human subjects. J Indian Med Assoc 107(6):403-405 
3. ClinicalTrials.gov (2020) Webpräsenz. https:// clinicaltrials.gov/ct2/home. Zugegriffen: 5. März 2020

4. Centre for Evidence Based Medicine (2020) Webpräsenz. https://www.cebm.net/. Zugegriffen: 7. Jan. 2020

5. Arunachalam S, Srinivasan R, Raman V (1994) International collaboration in science: participation by the Asian giants. Scientometrics 30:7-22

6. Autorino R, Quarto G, Di Lorenzo G et al (2007) Are abstracts presented at the EAU meeting followed by publication in peer-reviewed journals? A critical analysis. Eur Urol 51:833-840 (discussion 840)

7. Autorino R, Quarto G, Di Lorenzo G et al (2008) What happens to the abstracts presented at the Societè Internationale d'Urologie meeting? Urology 71:367-371

8. Autorino R, Quarto G, Sio MD et al (2006) Fate of abstracts presented at the World Congress of Endourology: Are they followed by publication in peer-reviewed journals? J Endourol 20:996-1001

9. Berthold F, Bartenhagen C, Krempel L (2019) Are network growth and the contributions to congresses associated with publication success? A pediatric oncology model. PLoSOne 14:e210994

10. Cartwright R, Khoo AK, Cardozo L (2007) Publish or be damned? The fate of abstracts presented at the International Continence Society Meeting 2003. Neurourol Urodyn 26:154-157

11. Castillo J, Garcia-Guasch R, Cifuentes I (2006) Fate of abstracts from the Paris 1995 European Society of Anaesthesiologists meeting. Eur J Anaesthesiol 19:888-893

12. Chen TJ, Chen YC, Hwang SJ et al (2007) International collaboration of clinical medicine research in Taiwan, 1990-2004: a bibliometric analysis. JChin Med Assoc 70:110-116

13. Chung JH, Autorino R, Kang DH et al (2012) Fate of abstracts presented at the annual meeting of the Korean Urological Association. Korean J Urol 53:280-284

14. Dal-ReR, Ross JS,MarusicA(2016)Compliancewith prospective trial registration guidance remained low in high-impact journals and has implications for primary end point reporting. J Clin Epidemiol 75:100-107

15. Hall KL, Vogel AL, Huang GC et al (2018) The science of team science: a review of the empirical evidence and research gaps on collaboration in science. Am Psychol 73:532-548

16. Hoag CC, Elterman DS, Macneily AE (2006) Abstracts presented at the American Urological Association Annual Meeting: determinants of subsequent peer reviewed publication. J Uro 176:2624-2629 (discussion 2629)

17. loannidis JP (2012) Are medical conferences useful? And for whom? JAMA 307:1257-1258

18. Ioannidis JPA (2018) Meta-research: why research on research matters. PLoSBiol 16:e2005468

19. Kunath F, Krause SF, Wullich B et al (2013) Bladder cancer-the neglected tumor: a descriptive analysis of publications referenced in MEDLINE and data from the register ClinicalTrials.gov. BMC Urol 13:56

20. Manolio TA, Bailey-Wilson JE, Collins FS (2006) Genes, environment and the value of prospective cohort studies. Nat Rev Genet 7:812-820

21. Ng L, Hersey K, Fleshner N (2004) Publication rate of abstracts presented at the annual meeting of the American Urological Association. BJU Int 94:79-81

22. Oliveira LR, Figueiredo AA, Choi Met al (2009) The publication rate of abstracts presented at the 2003 urological Brazilian meeting. Clinics (Sao Paulo) 64:345-349
23. Piwowar $\mathrm{H}$, Priem J, Larivière V et al (2018) The state of OA: a large-scale analysis of the prevalence and impact of Open Access articles. PeerJ 6:e4375

24. Rao AR, Beatty JD, Laniado $M$ et al (2006) Publication rate of abstracts presented at the British Association of Urological Surgeons Annual Meeting. BJU Int 97:306-309

25. Ross JS, Gross CP, Desai MM et al (2006) Effect of blinded peer review on abstract acceptance. JAMA 295:1675-1680

26. Scherer RW, Langenberg P, Von Elm E (2007) Full publication of results initially presented in abstracts. Cochrane Database Syst Rev 2:MR5

27. Scherer RW, Meerpohl JJ, Pfeifer N et al (2018) Full publication of results initially presented in abstracts. Cochrane Database Syst Rev 11:MR5

28. Shafer SL, Dexter F (2012) Publication bias, retrospective bias, and reproducibility of significant results in observational studies. Anesth Analg 114:931-932

29. Von Hardenberg J, Worst T, Weiss $C$ et al (2013) Abstracts at congresses of the German Society of Urology. Trends and quality. Urologe A 52:1296-1301

30. Weber WE, Merino JG, Loder E (2015) Trial registration 10 years on. BMJ 351:h3572

31. Yoon PD, Chalasani V, Woo HH (2012) Conversion rates of abstracts presented at the Urological Society of Australia and New Zealand (USANZ) Annual Scientific Meeting into full-text journal articles. BJU Int 110:485-489

32. Zhang C, Yu Q, Fan Q et al (2013) Research collaboration in health management research communities. BMC Med Inform Decis Mak 13:52

\section{Best-of DGU 2020}

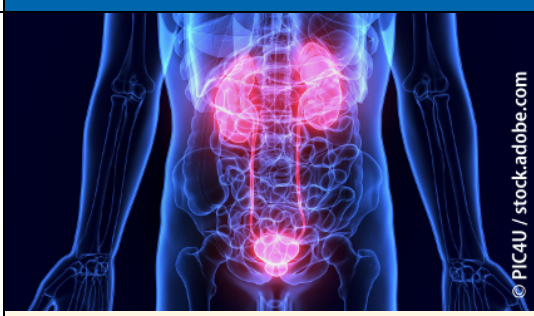

\section{UROLITHIASIS}

FREITAG, 25.09.2020

14:00 - 15:30 UHR

\section{MODERATION}

Knoll, T., Sindelfingen

Bach, T., Hamburg

Gierth, M., Regensburg

- 14:00 - Uro Dyna CT für alle oder doch IVP?: Wie entwickelt sich die Bildgebung in der Steintherapie? Ritter, M., Bonn

- 14:10 - Der neue Laser Goldstandard? Was kann ein Thuliumfaserlaser wirklich? Chun, F., Frankfurt

- 14:25 - The sky is no limit? Wo liegen die Grenzen der URS? Bach, T., Hamburg

- 14:35 - Diskussion

- 14:45 - Verleihung des Christian Chaussy-Award, Chaussy, C., Straßlach

- 14:50 - Der 15 mm Unterpolstein: Was tun? ESWL, Flexible URS, Mini PCNL, Neisius, A., Trier, Straub, M., München, Nagele, U., Hall, Österreich

- 15:10 - 50 Jahre Steintherapie: Was wurde erreicht (und was nicht)? Alken, P., Mannheim

- 15:20 - Diskussion und Fazit Bach, T., Hamburg

72. Kongress der Deutschen Gesellschaft für Urologie e.V. LIVE ONLINE.

Alle weiteren Informationen zur Registrierung finden Sie unter http://www.dgu-serviceforum.de/ egistrierung/teilnehmer/

Deutsche Gesellschaft für Urologie e.V. 